\title{
Modeling and Design Optimization of Energy Transfer Rate for Hybrid Energy Storage System in Electromagnetic Launch
}

\author{
Tao Ma, Junyong Lu *, Xiao Zhang, Bofeng Zhu, Wenxuan Wu and Xinlin Long
}

check for

updates

Citation: Ma, T.; Lu, J.; Zhang, X.; Zhu, B.; Wu, W.; Long, X. Modeling and Design Optimization of Energy Transfer Rate for Hybrid Energy Storage System in Electromagnetic Launch. Energies 2022, 15, 695. https://doi.org/10.3390/en15030695

Academic Editor: Andrea Mariscotti

Received: 17 August 2021

Accepted: 7 October 2021

Published: 18 January 2022

Publisher's Note: MDPI stays neutral with regard to jurisdictional claims in published maps and institutional affiliations.

Copyright: (c) 2022 by the authors. Licensee MDPI, Basel, Switzerland. This article is an open access article distributed under the terms and conditions of the Creative Commons Attribution (CC BY) license (https:// creativecommons.org/licenses/by/ $4.0 /)$.
National Key Laboratory of Science and Technology on Vessel Integrated Power System, Naval University of Engineering, Wuhan 430033, China; tao_ma@live.com (T.M.); xiao_zhang2019@163.com (X.Z.); bfzhu1990@163.com (B.Z.); chinawenxuanwu123@163.com (W.W.); xllong2019@163.com (X.L.)

* Correspondence: markbluesky@163.com

\begin{abstract}
The battery-pulse capacitor-based hybrid energy storage system has the advantage of high-energy density and high-power density. However, to achieve a higher firing rate of the electromagnetic launch, a shorter charging time of the pulse capacitor from the battery is needed. A new optimization model by formulating the charging time problem as a constrained optimization problem is presented. Unlike existing algorithms, the proposed model can find the globally optimal solution. The circuit parameters are optimized through the Enumeration algorithm to minimize the total charging time of the pulse capacitors from batteries. The simulation results show that the charging time of the proposed algorithm is shorter than the compared methods. Furthermore, a better solution could be obtained by canceling the constraint on the first peak of the charging current of the compared methods, which makes the circuit design more flexible for the hybrid energy storage system in the electromagnetic launch.
\end{abstract}

Keywords: electromagnetic launch; hybrid energy storage; power supply; sequential cascade control step-up charging; charging time; firing rate

\section{Introduction}

The electromagnetic launch is technology using the Lorentz force to accelerate an object to a super high speed, converting the electromagnetic energy to kinetic energy quickly [1-3]. To satisfy electromagnetic launch requirements, the pulsed power supplies should supply giga-watt-level power and mega-ampere-level current [4]. The pulsed power system plays a crucial role in the electromagnetic launch system. To date, there is much literature about pulsed power technologies with different technical approaches [4-10]. To meet both the high-energy density and high-power density requirements of the electrical pulse energy supply chain for the electromagnetic launch, a hybrid energy storage technology is widely utilized [2,11-15]. The most common scheme is the battery-pulse capacitor-based hybrid energy storage system [16-19]. However, to achieve a higher firing rate of the electromagnetic launch, a shorter charging time of the pulse capacitor from the battery is needed.

Improving the charging efficiency of the pulse capacitor from the battery is not a new problem, and many theoretical and experimental investigations have focused on the subject [20-22]. Li et al. [21] compared the three charging methods of direct current (DC) chopping charging, constant voltage charging, and sequential cascade control step-up charging, in detail, and the energy transfer rate of both DC chopping and the sequential cascade control step-up charging methods are higher than the constant voltage charging method. Furthermore, the sequential cascade control step-up charging method has lower requirements for switching devices and a simpler circuit than the DC chopping charging method. It is safer and more reliable. Hence, the sequential cascade control step-up charging method is more suitable for high-voltage, high-current, and large-scale energy 
storage working conditions. Long et al. [20] analyzed the charging process, based on the sequential cascade control step-up charging method, for a specific battery-pulse capacitorbased hybrid energy storage circuit and shortened the charging time by fine-tuning the battery cascading triggering time. Wu et al. [22] considered that the existing hybrid energy storage circuit is not optimal. They optimized the circuit by comparing the charging time of four circuits with the different values of resistance and inductance, where the resistance and inductance are constrained by each other according to the value of the first peak of the charging current.

In this paper, to improve the firing rate of the electromagnetic launch, we focus on shortening the charging time. Finding the optimal solutions to this charging time problem via the sequential cascade control step-up charging method in $[20,22]$ is not easy because the charging procedure is complex. It is difficult to obtain the analytical expression of the total charging time.

It is noticed that the methods mentioned above share some common points. Firstly, the research is aimed at a specific circuit, and all electrical parameters in the circuit are known. In order to achieve the purpose of optimization, it only makes some simple adjustments to these known parameters. Secondly, the first peak value of the charging current is constrained, which means that the resistance and inductance in the circuit are constrained by each other. However, for the constraint condition that the first peak value of the charging current reaches the specified value is unreasonable. It limits the optimal design of the circuit and is unable to obtain a better solution.

This paper presents a novel optimization model by formulating the charging time problem as a constrained optimization problem. The constraint on the first peak of the charging current is canceled. The electrical parameters of the research circuit are unknown. This innovative model can find the globally optimal solution through the Enumeration algorithm and get the minimum total charging time of pulse capacitors from batteries. The results show that the proposed algorithm performs better than the compared algorithms. It makes the circuit design more flexible for the hybrid energy storage system in the electromagnetic launch.

\section{Problem Formulation}

\subsection{Basic Circuit}

The battery-pulse capacitor-based hybrid energy storage system's charging circuit comprises the battery pack, the control switch, a current-limiting inductor, and the pulse capacitor. The battery pack includes lithium-ion cells arranged in series and parallel to provide the required charging voltage and current. In the charging process of the pulse capacitor from the battery, battery packs are connected to the charging circuit in series by utilizing the control switch to be turned on at different times. In this way, the output charging voltage can increase gradually. Meanwhile, the charging current is limited by the current-limiting inductor, causing the charging circuit to charge the capacitor with an approximately constant current.

The basic circuit is shown in Figure 1 , where $E_{1}-E_{N}$ are $N$ battery packs. Each battery pack consists of $n_{s}$ battery cells connected in series, and $n_{p}$ in parallel. $r_{e}$ is the internal resistance of a single battery cell. $r_{1}=r_{2}=\ldots=r_{N}=n_{s} / n_{p} \cdot r_{e}$ are the internal resistances of each battery pack. $R_{0 p}$ and $R_{0 n}$ are the resistances of the positive and negative transmission cables of the main circuit, respectively. $L_{0}$ is the stray inductance of the main circuit. $L$ and $R_{l}$ are the inductance and resistance of the current-limiting inductor, respectively. $C$ is the pulse capacitor. $D$ is the freewheeling diode. $K_{1}-K_{N}$ are control switches. IGBT, $R_{I}$, and $D_{I}$ together compose the main switch $K_{c}$ of the primary circuit.

Firstly, turn on the main switch $K_{c}$ and switch $K_{1}$ to connect the battery pack $E_{1}$ to the circuit. The first battery pack $E_{1}$ starts charging the pulse capacitor $C$, and both the voltage of $C$ and the charging current increase gradually. The charging current will decrease when the voltage of $C$ is close to the voltage of $E_{1}$. At this point, turn off $K_{1}$ and turn on $K_{2}$ to connect the second battery pack $E_{2}$ to the circuit, making $E_{1}$ and $E_{2}$ connected in series, 
and the charging current increases again until the voltage of $C$ is close to the voltage of $E_{1}$ and $E_{2}$ in series. Then, connect the next battery pack to the circuit, and so on, until the last battery pack, $E_{N}$, is connected to the circuit. Turn off the main switch $K_{c}$ when the voltage of $C$ reaches the required value. At this time, the circuit enters the freewheeling process. The freewheeling diode $D$, current-limiting inductor $L$, and capacitors $C$ constitute the freewheeling circuit. The current in the freewheeling circuit gradually decreases to zero. Eventually, the freewheeling diode $D$ is turned off, and the energy transfer process is over.

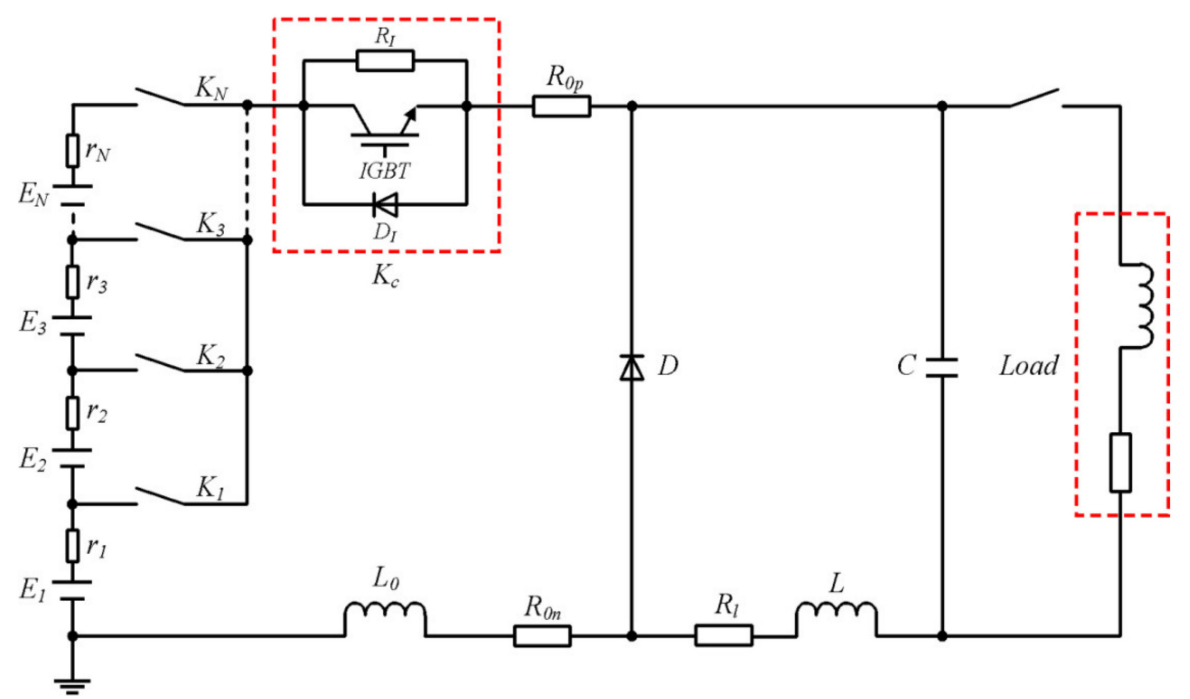

Figure 1. Diagram of the simplified circuit of the battery-pulse capacitor-based hybrid energy storage system.

\subsection{Energy Transfer Process}

2.2.1. Charging Process Analysis

Because the resistances of transmission cables and the stray inductance of the circuit are much smaller than those of the current-limiting inductor, they can be ignored in the following analysis. The simplified circuit is shown in Figure 2. Then, the charging process can be further simplified as follows:

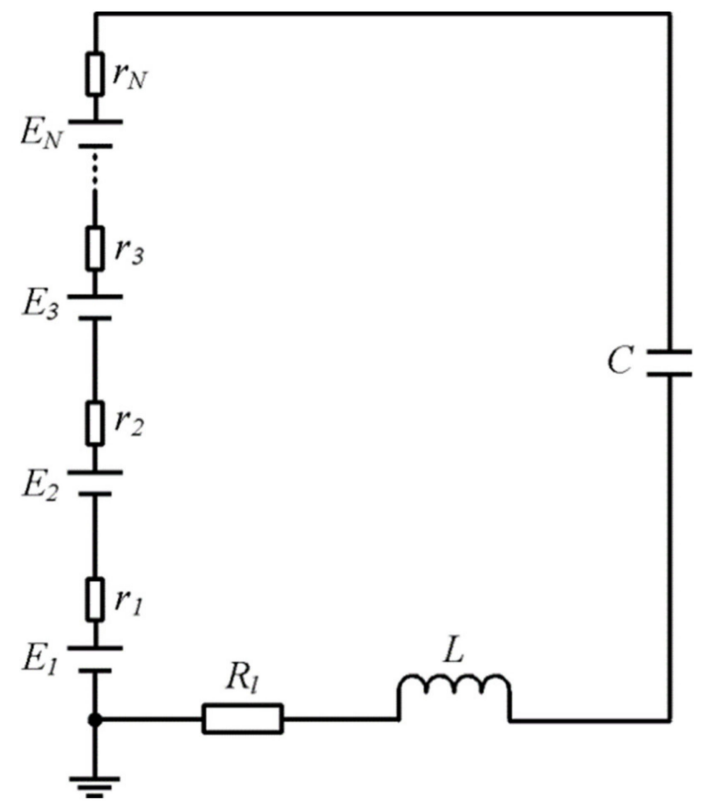

Figure 2. Diagram of the simplified circuit of the battery-pulse capacitor charging process. 
Note that the battery packs are connected to the charging circuit in series, one by one, at different times. The trigger time of the control switch is crucial. If the control switches are triggered in the proper time sequence, the charging current could be approximately constant. Generally, the constant value is designed to be the maximum charging current that can be reached. If a switch is triggered early, the following peak current will exceed the maximum current. If it is triggered late, the following peak current will not reach the required value. The waveform of the charging current is shown in Figure 3.

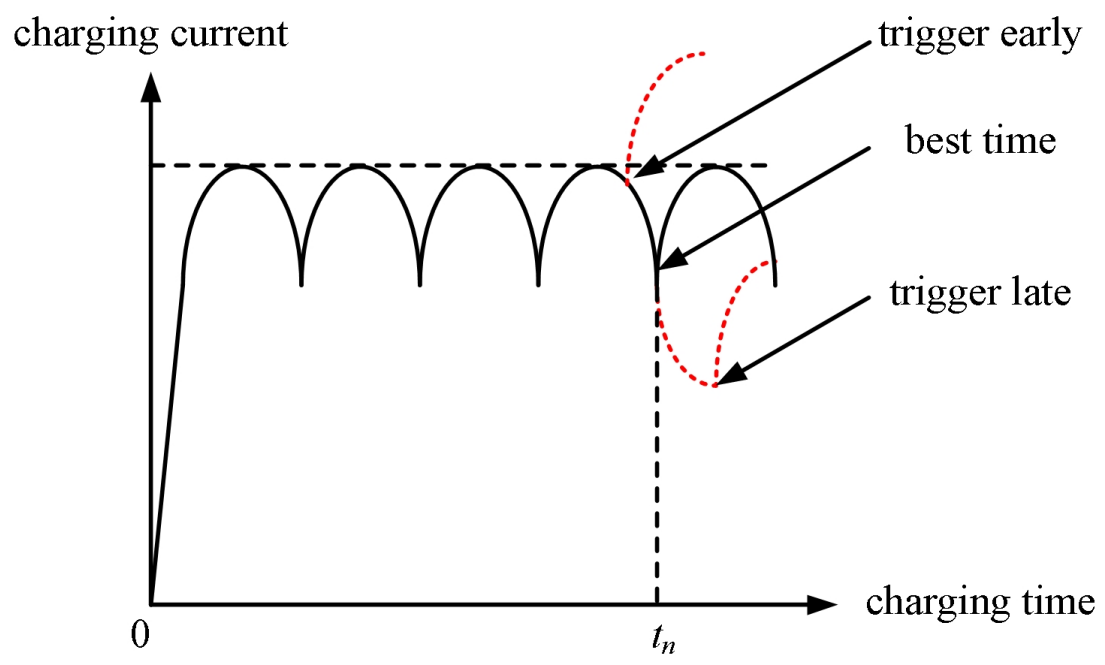

Figure 3. The charging current in the charging process.

In the battery-pulse capacitor-based hybrid energy storage system circuit in Figure 2, when the $n$th $(n=1,2, \ldots, N)$ battery pack $E_{n}$ is connected to the circuit, the voltage balance equation can be obtained by utilizing Kirchhoff's voltage law:

$$
R_{n} I(t)+L \frac{d I(t)}{d t}+V\left(t_{n}\right)+\frac{1}{C} \int_{t_{n}}^{t} I(t) d t-n V_{E}=0
$$

where $R_{n}=\left(r_{1}+r_{2}+\ldots+r_{n}\right)+R_{l}=n r_{1}+R_{l}=n n_{s} r_{e} / n_{p}+R_{l}$ is the total resistance in the circuit; $I(t)$ is the charging current; $v_{e}$ is the open-circuit voltage of each battery cell; $V_{E}=n_{s} v_{e}$ is the open-circuit voltage of each battery pack; and $V\left(t_{n}\right)$ is the voltage of the pulse capacitor at the time $t_{n}$ when the $n$th battery pack $E_{n}$ is connected to the circuit.

Performing the derivation operation with respect to $I(t)$ on both sides of Equation (1), we obtain

$$
\frac{d^{2} I(t)}{d t^{2}}+\frac{R_{n}}{L} \frac{d I(t)}{d t}+\frac{1}{C L} I(t)=0
$$

The system satisfies the underdamped oscillating circuit condition when $R_{n}<2 \sqrt{L / C}$. Then, solve the above second-order linear homogeneous differential Equation (2) yields:

$$
\begin{gathered}
V(t)=e^{\alpha\left(t-t_{n}\right)}\left[k_{1} \cos \beta\left(t-t_{n}\right)+k_{2} \sin \beta\left(t-t_{n}\right)\right]+n V_{E} \\
I(t)=C e^{\alpha\left(t-t_{n}\right)}\left[\left(\alpha k_{1}+\beta k_{2}\right) \cos \left(\beta\left(t-t_{n}\right)\right)\right. \\
\left.+\left(\alpha k_{2}-\beta k_{1}\right) \sin \left(\beta\left(t-t_{n}\right)\right)\right]
\end{gathered}
$$

and the time constant $\tau_{1}$

$$
\tau_{1}=\frac{2 L}{R_{n}}
$$

with

$$
\alpha=-\frac{R_{n}}{2 L}
$$




$$
\begin{gathered}
\beta=\sqrt{\frac{1}{L C}-\frac{R_{n}^{2}}{4 L^{2}}} \\
k_{1}=-n V_{E}+V\left(t_{n}\right) \\
k_{2}=\frac{1}{\beta}\left[\frac{I\left(t_{n}\right)}{C}-k_{1} \alpha\right]
\end{gathered}
$$

Let $d I(t) / d t=0$. We can obtain the maximum value of $I(t)$ after the $n$th battery pack $E_{n}$ is connected to the circuit and time $t_{m}$ at this moment. That is,

$$
\begin{aligned}
t_{m}(n)= & \frac{1}{\beta} \arctan \frac{\left(\alpha^{2}-\beta^{2}\right) k_{1}+2 \alpha \beta k_{2}}{\left(\beta^{2}-\alpha^{2}\right) k_{2}+2 \alpha \beta k_{1}}+t_{n}, n=1,2, \ldots N \\
I_{m}(n)= & C e^{\alpha\left(t_{m}(n)-t_{n}\right)}\left[\left(\alpha k_{1}+\beta k_{2}\right) \cos \left(\beta\left(t_{m}(n)-t_{n}\right)\right)\right. \\
& \left.+\left(\alpha k_{2}-\beta k_{1}\right) \sin \left(\beta\left(t_{m}(n)-t_{n}\right)\right)\right], n=1,2, \ldots N
\end{aligned}
$$

Assume that $I_{M}$ is the required maximum charging current. If $I_{m}>I_{M}$, move the trigger time $t_{n}$ backward. Otherwise, move the trigger time $t_{n}$ forward. Adjust the trigger time $t_{n}$ until $I_{m}$ is equal to $I_{M}$, and connect the battery pack $E_{n}$ to the circuit. Then consider the next battery pack, $E_{n+1}$, and so on, until the pulse capacitor is charged to the specified voltage $V_{M}$.

\subsubsection{Freewheeling Process Analysis}

The main switch, $K_{c}$, will be turned off when the voltage of $C$ reaches the required value. The circuit enters the freewheeling process. The energy stored in the current-limiting inductor $L$ will continue to be transferred to the pulse capacitor $C$ until the current decreases to zero. Then the diode $D$ is turned off. The freewheeling circuit is shown in Figure 4.

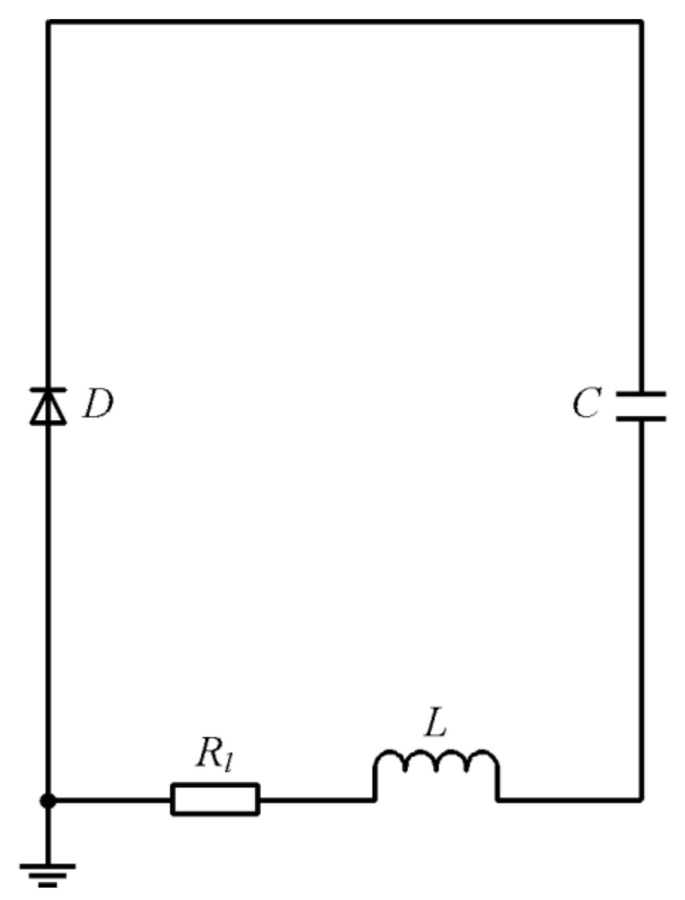

Figure 4. Diagram of the simplified circuit of the battery-pulse capacitor freewheeling process.

According to Kirchhoff's voltage law, we can obtain the voltage balance equation:

$$
R_{l} I(t)+V\left(t_{c}\right)+L \frac{d I(t)}{d t}+\frac{1}{C} \int_{t_{c}}^{t} I(t) d t=0
$$


where $t_{c}$ is the turn-off time of the main switch $K_{c}$. Performing the derivation operation with respect to $I(t)$ on both sides of Equation (8), we can get

$$
\frac{d^{2} I(t)}{d t}+\frac{R_{l}}{L} \frac{d I(t)}{d t}+\frac{1}{C L} I(t)=0
$$

$R_{l}<2 \sqrt{L / C}$, so that the circuit satisfies the underdamped condition. Then, solving Equation (9) yields

$$
\begin{aligned}
V(t)= & e^{A\left(t-t_{c}\right)}\left[k_{3} \cos B\left(t-t_{c}\right)+k_{4} \sin B\left(t-t_{c}\right)\right] \\
I(t)= & C e^{A\left(t-t_{c}\right)}\left[\left(A k_{3}+B k_{4}\right) \cos \left(B\left(t-t_{c}\right)\right)\right. \\
& \left.+\left(A k_{4}-B k_{3}\right) \sin \left(B\left(t-t_{c}\right)\right)\right]
\end{aligned}
$$

and the time constant $\tau_{2}$

$$
\tau_{2}=\frac{2 L}{R_{l}}
$$

with

$$
\begin{gathered}
A=-\frac{R_{l}}{2 L} \\
B=\sqrt{\frac{1}{L C}-\frac{R_{l}^{2}}{4 L^{2}}} \\
k_{3}=V\left(t_{c}\right) \\
k_{4}=\frac{1}{B}\left[\frac{I\left(t_{c}\right)}{C}-k_{3} A\right]
\end{gathered}
$$

Let $I(t)=0$. We can obtain the time $t_{\text {tot }}$ when the freewheeling process is finished.

$$
t_{\text {tot }}=\frac{1}{B} \arctan \frac{k_{3} A+k_{4} B}{k_{3} B-k_{4} A}+t_{c}
$$

That is, $t_{\text {tot }}$ is the total charging time of the battery-pulse capacitor-based hybrid energy storage system.

\subsection{Principle of Wu's Algorithm}

\subsubsection{Known Conditions Description}

The research in the paper [22] is for a specific circuit. All electrical parameters in the hybrid energy storage circuit are known. They are listed in Table 1.

Wu et al. considered that the existing hybrid energy storage circuit is not optimal and optimized the circuit by adjusting the resistance value of the circuit. Three different adjustments are considered, that is,

$$
\begin{aligned}
& R_{l 1}=75 \% \times R_{l} \\
& R_{l 2}=50 \% \times R_{l} \\
& R_{l 3}=25 \% \times R_{l}
\end{aligned}
$$

Furthermore, the first peak value of the charging current is constrained, that is, $I_{m}(1)=I_{M}$. It causes the resistance and inductance in the circuit to be constrained by each other. Then, Formulas (6) and (7) can be rewritten as:

$$
t_{m}(1)=\frac{1}{\beta} \arctan \frac{\left(\alpha^{2}-\beta^{2}\right) k_{1}+2 \alpha \beta k_{2}}{\left(\beta^{2}-\alpha^{2}\right) k_{2}+2 \alpha \beta k_{1}}
$$




$$
\begin{aligned}
& I_{m}(1)=I_{M} \\
& =C e^{\alpha t_{m}(1)}\left[\left(\alpha k_{1}+\beta k_{2}\right) \cos \left(\beta\left(t_{m}(1)\right)\right)\right. \\
& \left.+\left(\alpha k_{2}-\beta k_{1}\right) \sin \left(\beta\left(t_{m}(1)\right)\right)\right]
\end{aligned}
$$

Table 1. The states of electrical parameters in the hybrid energy storage circuit before the optimization of Wu's algorithm.

\begin{tabular}{ccc}
\hline Parameter & Description & State \\
\hline$C$ & the capacitance of pulse capacitor & known \\
$V_{M}$ & required charging voltage & known \\
$v_{e}$ & the open-circuit voltage of each battery cell & known \\
$r_{e}$ & the internal resistance of each battery cell & known \\
$I_{e}$ & the maximum continuous discharge current of each battery cell & known \\
$I_{M}$ & required maximum charging current & known \\
$I_{c}$ & maximum turn-off current of the main switch $K_{c}$ & known \\
$n_{S}$ & number of series batteries in each battery pack & known \\
$n_{p}$ & number of parallel batteries in each battery pack & known \\
$N$ & total number of battery packs & known \\
$n_{t o t}=N n_{s} n_{p}$ & total number of battery cells & known \\
$V_{E}=n_{s} v_{e}$ & the open-circuit voltage of each battery pack & known \\
$r_{n}=n_{s} r_{e} / n_{p}$ & the internal resistance of each battery pack & known \\
$L$ & the inductance of the current-limiting inductor & known \\
$R_{l}$ & the resistance of the current-limiting inductor & known \\
\hline
\end{tabular}

Calculate inductance values $\left(L, L_{1}, L_{2}, L_{3}\right)$ for different resistance values $\left(R_{l}, R_{l 1}, R_{l 2}, R_{l 3}\right)$ using Formulas (15) and (16), respectively. Then, four groups of parameters can be obtained for the comparison:

$$
\begin{array}{llll}
\text { Group } 1: & \left(L, R_{l}\right), & \text { Group } 2: & \left(L_{1}, R_{l 1}\right), \\
\text { Group } 3: & \left(L_{2}, R_{l 2}\right), & \text { Group } 4: & \left(L_{3}, R_{l 3}\right) .
\end{array}
$$

\subsubsection{Algorithm Description}

The procedures of calculating the total charging time $t_{\text {tot }}$ are summarized as follows:

(Step 1): Initialize the parameters of the algorithms in Table 1. Select a group of values from (17) for the resistance and inductance in the circuit.

(Step 2): Set the number of battery packs connected to the circuit $n=1$, the trigger time $t_{n}=0$, initial voltage $V\left(t_{n}\right)=0$, and initial current $I\left(t_{n}\right)=0$.

(Step 3): Calculate $t_{m}(n), I_{m}(n)$, and $V\left(t_{m}(n)\right)$ according to Formulas (6), (7) and (3), respectively, after the $n$th battery pack $E_{n}$ is connected to the circuit. Set the trigger time $t_{n+1}=t_{m}(n)$ for the $(n+1)$ th battery pack $E_{n+1}$ to be connected.

(Step 4): Calculate $V\left(t_{n+1}\right)$ and $I\left(t_{n+1}\right)$ according to Formulas (3) and (4). Then, calculate $t_{m}(n+1), I_{m}(n+1)$, and $V\left(t_{m}(n+1)\right)$ according to Formulas (6), (7) and (3) for the circuit calculation of the following battery pack $E_{n+1}$ to be connected.

(Step 5): If $I_{m}(n+1)>I_{M}, t_{n+1}=t_{n+1}+0.001$, go to (Step 4); otherwise, $n=n+1$.

(Step 6): If $n<N$, go to (Step 3); otherwise, calculate the $V\left(t_{n}\right)$ and $I\left(t_{n}\right)$ according to Formulas (3) and (4).

(Step 7): If $V\left(t_{n}\right)<V_{M}, t_{n}=t_{n}+0.001$ go to (Step 6); otherwise, $t_{c}=t_{n}, V\left(t_{c}\right)=V\left(t_{n}\right)$, and $I\left(t_{c}\right)=I\left(t_{n}\right)$.

(Step 8): Using the Formula (13), the total charging time $t_{\text {tot }}$ can be obtained.

For the four groups of different parameters in (17), four total charging times can be obtained through the above calculation process. Among them, the parameter corresponding to the shortest total charging time is considered the final result of the optimization of $\mathrm{Wu}^{\prime} \mathrm{s}$ algorithm. The flow chart of the procedures of calculating the total charging time is shown in Figure 5. 


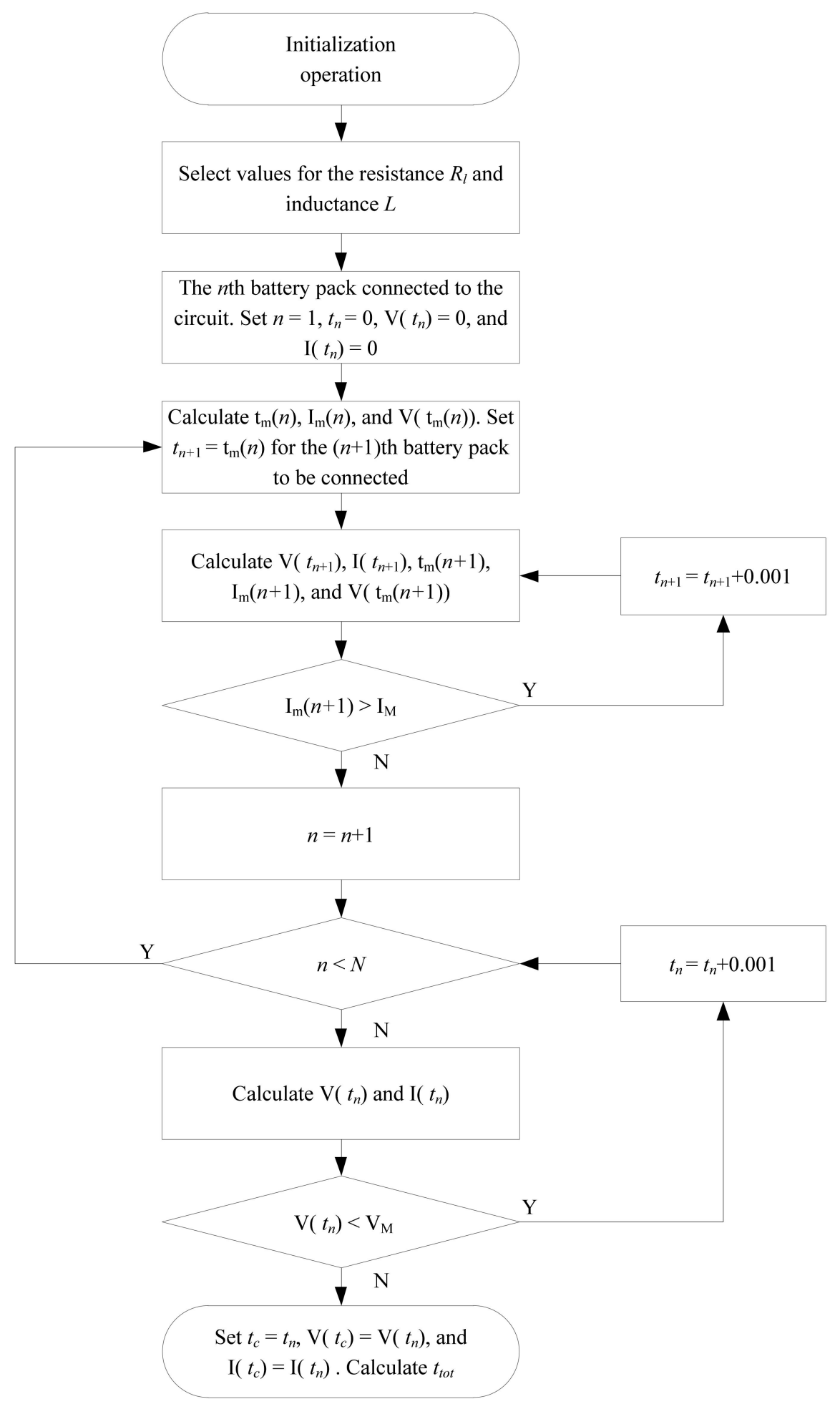

Figure 5. The flow chart of the procedures for calculating the total charging time.

\section{Methodology}

\subsection{Known Conditions Description}

Compared with the specific circuit studied in [22], we face an unknown circuit that needs to be designed in practical applications. In this paper, the states of the electrical parameters in the circuit before the optimization are listed in Table 2. 
Table 2. The states of electrical parameters in the hybrid energy storage circuit before the optimization of the Enumeration algorithm.

\begin{tabular}{ccc}
\hline Parameter & Description & State \\
\hline$C$ & the capacitance of pulse capacitor & known \\
$V_{M}$ & required charging voltage & known \\
$v_{e}$ & the open-circuit voltage of each battery cell & known \\
$r_{e}$ & the internal resistance of each battery cell & known \\
$I_{e}$ & the maximum continuous discharge current of each battery cell & known \\
$I_{M}$ & required maximum charging current & known \\
$I_{c}$ & maximum turn-off current of the main switch $K_{c}$ & known \\
$n_{S}$ & number of series batteries in each battery pack & unknown \\
$n_{p}$ & number of parallel batteries in each battery pack & unknown \\
$N$ & total number of battery packs & unknown \\
$n_{t o t}=N n_{s} n_{p}$ & total number of battery cells & known \\
$V_{E}=n_{s} v_{e}$ & the open-circuit voltage of each battery pack & unknown \\
$r_{n}=n_{s} r_{e} / n_{p}$ & the internal resistance of each battery pack & unknown \\
$L$ & the inductance of the current-limiting inductor & unknown \\
$R_{l}$ & the resistance of the current-limiting inductor & unknown \\
\hline
\end{tabular}

As shown in Table 2, there are seven unknown parameters. In fact, there are only five unknown parameters. Because the open-circuit voltage of each battery pack $V_{E}=n_{s} v_{e}$ is the function of $n_{s}$, and the internal resistance of each battery pack $r_{n}=n_{s} r_{e} / n_{p}$ is the function of $n_{s}$ and $n_{p}$, both $v_{e}$ and $r_{e}$ are known.

It can be seen from the table that the structure of a single battery pack, the total number of battery packs, and the inductance and resistance of the circuit are all unknown and need to be designed. However, none of these questions are answered in the existing research.

\subsection{Mathematical Model}

A novel optimization model by formulating the charging time problem as a constrained optimization problem is presented in this paper. The constraint on the first peak of the charging current is canceled.

\subsubsection{Optimization Objective Function}

The objective function is as follows:

$$
\text { minimize } f(\mathbf{x})=t_{\text {tot }}(\mathbf{x})
$$

with

$$
\begin{aligned}
\mathbf{x} & =\left[\begin{array}{lllll}
n_{s} & n_{p} & N & L & R_{l}
\end{array}\right]^{T} \\
& =\left[\begin{array}{lllll}
x_{1} & x_{2} & x_{3} & x_{4} & x_{5}
\end{array}\right]^{T}
\end{aligned}
$$

\subsubsection{Constraint Condition}

Constraints of functions are shown in the following formulas:

$$
\begin{gathered}
g_{1}(\mathbf{x})=n_{s} \cdot n_{p} \cdot N-n_{t o t} \leq 0 \\
g_{2}(\mathbf{x})=V_{M}-n_{s} \cdot N \cdot v_{e}<0 \\
g_{3}(\mathbf{x})=I_{M}-n_{p} \cdot I_{e} \leq 0 \\
g_{4}(\mathbf{x})=n_{p} \cdot I_{e}-I_{c}<0 \\
g_{5}(\mathbf{x})=I_{m}(n)-I_{M} \leq 0, \quad n=1 \\
g_{5}(\mathbf{x})=I_{m}(n)-I_{M}=0, \quad n=2,3, \ldots N \\
g_{6}(\mathbf{x})=R_{N}-2 \sqrt{L / C}<0
\end{gathered}
$$




$$
\begin{gathered}
g_{7}(\mathbf{x})=L_{\min }-L \leq 0 \\
g_{8}(\mathbf{x})=L-L_{\max } \leq 0 \\
g_{9}(\mathbf{x})=R_{\min }-R_{l} \leq 0 \\
g_{10}(\mathbf{x})=R_{l}-R_{\max } \leq 0
\end{gathered}
$$

Formula (19) is the constraint that the number of battery cells to be used cannot exceed the total number of existing battery cells.

Formula (20) is the constraint that the total open-circuit voltage of all battery packs connected in series should be higher than the required charging voltage.

Formula (21) is the constraint that each battery pack's maximum continuous discharge current should be higher than the required maximum charging current.

Formula (22) is the constraint that each battery pack's maximum continuous discharge current should be lower than the maximum turn-off current of the main switch for safety reasons.

Formula (23) is the constraint of the peak of the charging current. The constraint $I_{m}(1)=I_{M}$ on the first peak of the charging current is canceled.

Formula (24) is the constraint of the underdamped oscillating circuit condition.

Formula (25) is the value ranges constraint of the resistance and inductance in the circuit. It mainly depends on the design of the current-limiting inductor.

Hence, the mathematical model by formulating the charging time problem as a constrained optimization problem can be described as follows:

$$
\begin{array}{lll}
\text { minimize } & f(\mathbf{x})=t_{\text {tot }}(\mathbf{x}) & \\
\text { subjectto } & \mathbf{x} \in \mathbb{R}^{5}, x_{i} \in \mathbb{Z}^{+}, & \text {for } i=1,2,3 \\
& g_{j}(\mathbf{x}) \leq 0, & \text { for } j=1,3,7,8,9,10 \\
& g_{j}(\mathbf{x})<0, \quad \text { for } j=2,4,6 \\
& g_{j}(\mathbf{x}) \leq 0, \quad \text { for } j=5, n=1 \\
& g_{j}(\mathbf{x})=0, \quad \text { for } j=5, n=2,3, \ldots N .
\end{array}
$$

\subsection{Enumeration Algorithm}

Note that the first three variables in the variable vector $\mathbf{x}$ are positive integers. Hence, the search space of the variable vectors would not be too large, and the Enumeration algorithm is suitable for solving this optimization problem.

Furthermore, our algorithm is divided into two parts: the variable selection and total charging time calculations and comparisons to reduce the computing time.

\subsubsection{Variable Selection}

The variable selection is aimed to get variable vectors that satisfy all constraints, and the procedures are given as follows:

(Step 1): Initialize the parameters in Table 2.

(Step 2): Select a variable vector $\mathbf{x}_{i}$ from the search space. Calculate $g_{1}\left(\mathbf{x}_{i}\right) \sim g_{10}\left(\mathbf{x}_{i}\right)$ using Formulas (19) (25). If all the constraints are satisfied, save this variable vector $\mathbf{x}_{i}$ in $\mathbf{X}$ and $i=i+1$; otherwise, $i=i+1$.

(Step 3): Repeat (Step 2) until the algorithm enumerates all the variable vectors in the search space. Then, $\mathbf{X}$ is the feasible solution set of the Enumeration algorithm.

The flow chart of the procedures of the variable selection is shown in Figure 6.

\subsubsection{Total Charging Time Calculation and Comparison}

The procedures for the total charging time calculation and comparison are summarized as follows:

(Step 1): Select a variable vector $\mathbf{x}_{j}$ in $\mathbf{X}$. Use the algorithm described in Section 2.3.2 to calculate the total charging time $t_{\text {tot }}\left(\mathbf{x}_{j}\right)$ and save it. Then $j=j+1$. 
(Step 2): Repeat (Step 1) until the algorithm enumerates all the variable vectors in $\mathbf{X}$.

(Step 3): Comparing all the total charging time calculation results, select the variable vector $\mathbf{x}^{*}$ corresponding to the minimum value as the optimization result of the Enumeration algorithm.

The flow chart of the procedures of the total charging time calculation and comparison is shown in Figure 7.

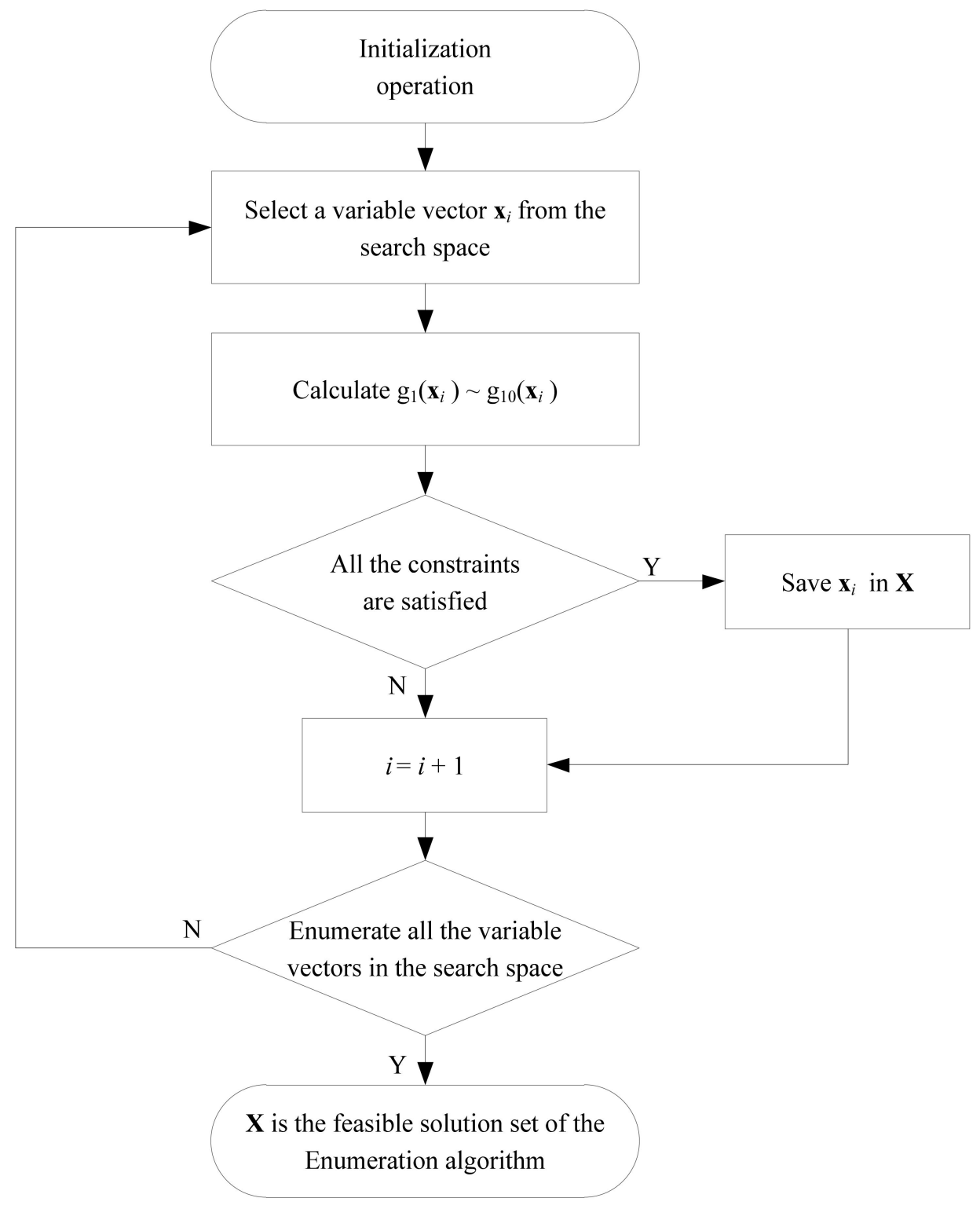

Figure 6. The flow chart of the procedures for the variable selection. 


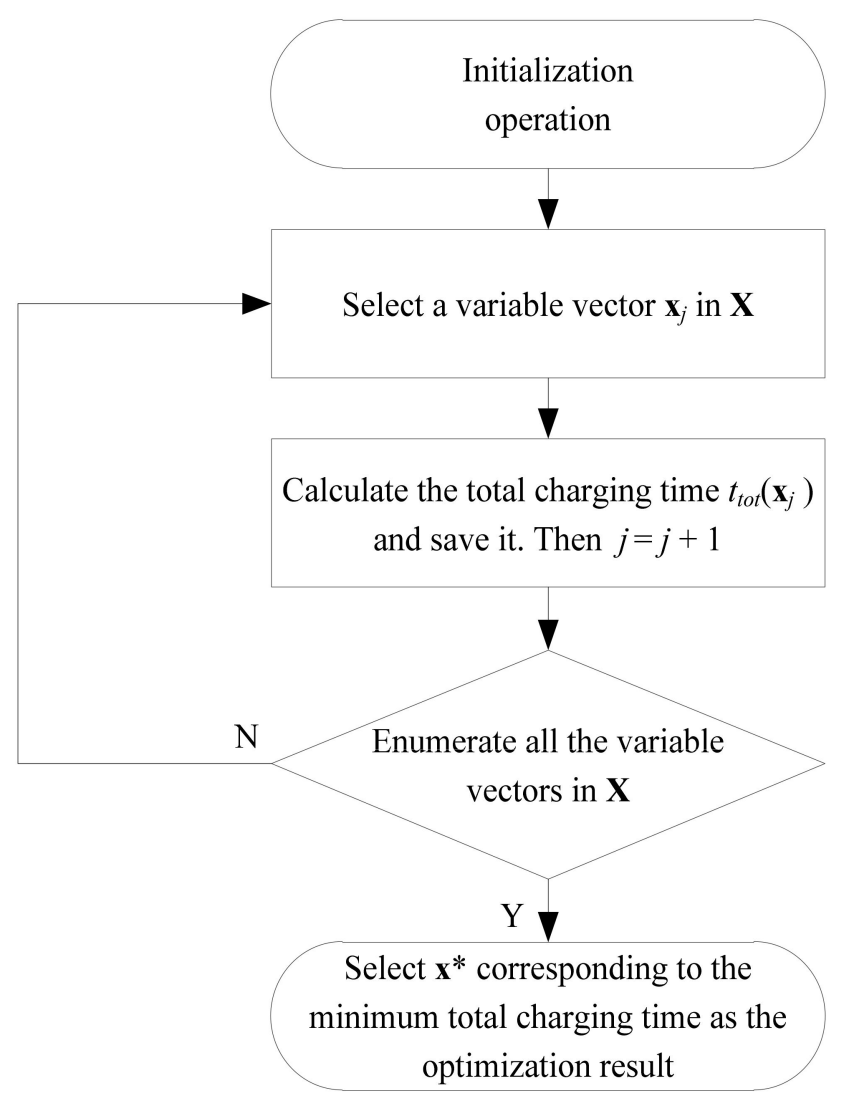

Figure 7. The flow chart of the procedures for the total charging time calculation and comparison.

\section{Simulation Studies and Analysis}

In this section, simulations have been made to assess the performance of the proposed Enumeration algorithm and compare it with $\mathrm{Wu}$ 's algorithm.

To have a better comparison, we set the basic circuit parameters to be the same as those in [22], and they are presented in Table 3.

Table 3. The primary electrical parameters in the hybrid energy storage circuit in the simulation studies.

\begin{tabular}{ccccccc}
\hline Parameter & $C(\mathrm{~F})$ & $v_{e}(\mathrm{~V})$ & $r_{e}(\mathrm{~m} \Omega)$ & $I_{e}(\mathrm{~A})$ & $I_{M}(\mathrm{~A})$ & $I_{c}(\mathrm{~A})$ \\
\hline Value & 0.5 & 3.2 & 1.97 & 250 & 1250 & 1300 \\
\hline
\end{tabular}

The value ranges of the inductance $L$ and resistance $R_{l}$ in the circuit are set to $[5 \mathrm{mH}, 40 \mathrm{mH}]$ and $[30 \mathrm{~m} \Omega, 200 \mathrm{~m} \Omega]$, respectively.

Two different cases are compared. The required charging voltage and the total number of battery cells of both cases are shown in Table 4 .

Table 4. The required charging voltage and the total number of battery cells of two cases.

\begin{tabular}{ccc}
\hline & $V_{M}(\mathbf{V})$ & $n_{\text {tot }}$ \\
\hline Case 1 & 4300 & 6875 \\
Case 2 & 5500 & 8750 \\
\hline
\end{tabular}

\subsection{Case 1}

First, let us consider the variable selection results and the corresponding total charging time calculation results of the Enumeration algorithm. Figure 8 shows the total charging time calculation results corresponding to all the variable vectors in $\mathbf{X}$. There are 
4307 solutions in total. Among these points, the optimal solution is associated with the minimum total charging time, shown by the pentagram.

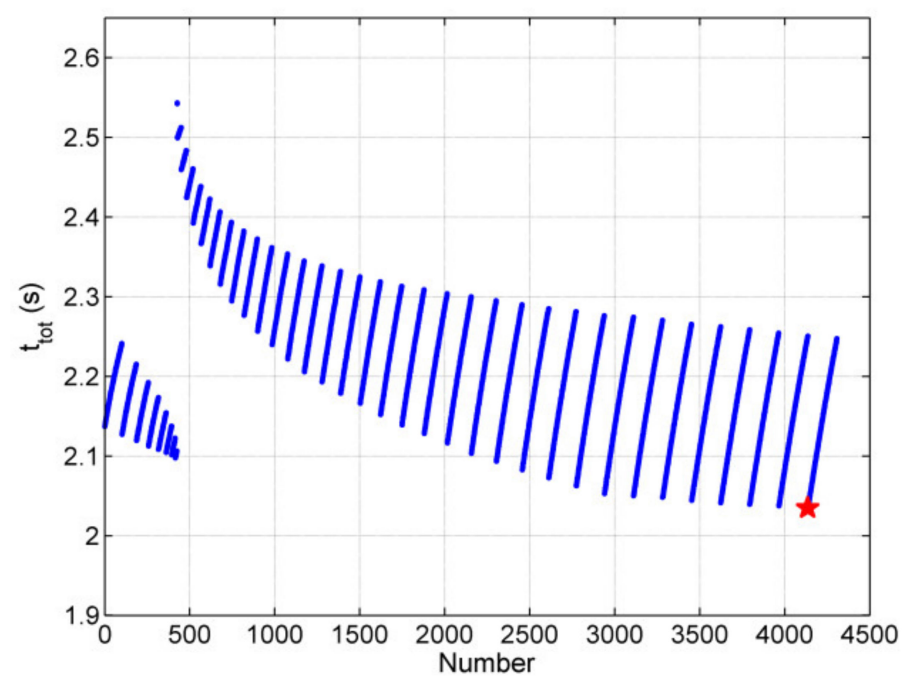

Figure 8. Calculation results of total charging time corresponding to all the variable vectors in $\mathbf{X}$.

From this figure, we can see that the results consist of two parts. The two parts correspond to two battery pack structures, respectively. Table 5 shows the details of the two battery pack structures.

Table 5. Two battery pack structures in the solutions.

\begin{tabular}{cccccc}
\hline Condition & $n_{\boldsymbol{s}}$ & $n_{\boldsymbol{p}}$ & $\boldsymbol{N}$ & Number & $\min \left(\boldsymbol{t}_{\text {tot }}\right)(\mathrm{s})$ \\
\hline 1 & 55 & 5 & 25 & 425 & 2.10 \\
2 & 125 & 5 & 11 & 3882 & 2.03 \\
\hline
\end{tabular}

The optimal solutions of Wu's algorithm and the Enumeration algorithm are listed in Table 6. From the table, we can see that the total charging time of the Enumeration algorithm is slightly shorter than that of Wu's algorithm, but not significantly. The optimal solutions of both algorithms are better than that of the solution before the optimization. It is noted that "before the optimization" means the algorithm before the optimization of $\mathrm{Wu}$ 's algorithm. It refers to the initial state of the existing hybrid energy storage circuit with the first peak value of the charging current is constrained. In other words, it is just an ordinary feasible solution that satisfies the constraints condition $I_{m}(1)=I_{M}$.

Table 6. Comparison results of the two algorithms.

\begin{tabular}{ccccccc}
\hline & $n_{\boldsymbol{s}}$ & $n_{\boldsymbol{p}}$ & $\boldsymbol{N}$ & $\boldsymbol{L}(\mathbf{m H})$ & $\boldsymbol{R}_{\boldsymbol{l}}(\mathbf{m} \Omega)$ & $\min \left(\boldsymbol{t}_{\text {tot }}\right)(\mathrm{s})$ \\
\hline Before the optimization & 125 & 5 & 11 & 11.5 & 152 & 2.38 \\
Wu's algorithm & 125 & 5 & 11 & 31.2 & 37 & 2.06 \\
Enumeration algorithm & 125 & 5 & 11 & 40 & 30 & 2.03
\end{tabular}

Note that the battery pack structure of the specific circuit that Wu's algorithm aims at is the same as that in the optimal solution of the Enumeration algorithm. However, the solving process is unknown. Thus, we can see that the specific circuit has been optimized before using Wu's algorithm to optimize, but the optimization process is unknown.

The comparison of the charging current-time and charging voltage-time curves of the pulse capacitor of the two algorithms are presented in Figures 9 and 10, respectively. As shown in Figure 9, unlike Wu's algorithm, the first peak value of the charging current of the Enumeration algorithm is $1155 \mathrm{~A}$, and does not reach the required maximum charging 
current 1250 A. Furthermore, except for the first peak value, all the other peak values reach the required maximum charging current. The charging current of the Enumeration algorithm has more minor fluctuations and is closer to a constant value. These results indicate that the constraint condition that the first peak value of the charging current needs to reach a specified value is unreasonable. It limits the optimal design of the circuit. Specifically, it limits the design of the current-limiting inductor and is unable to obtain a better solution.

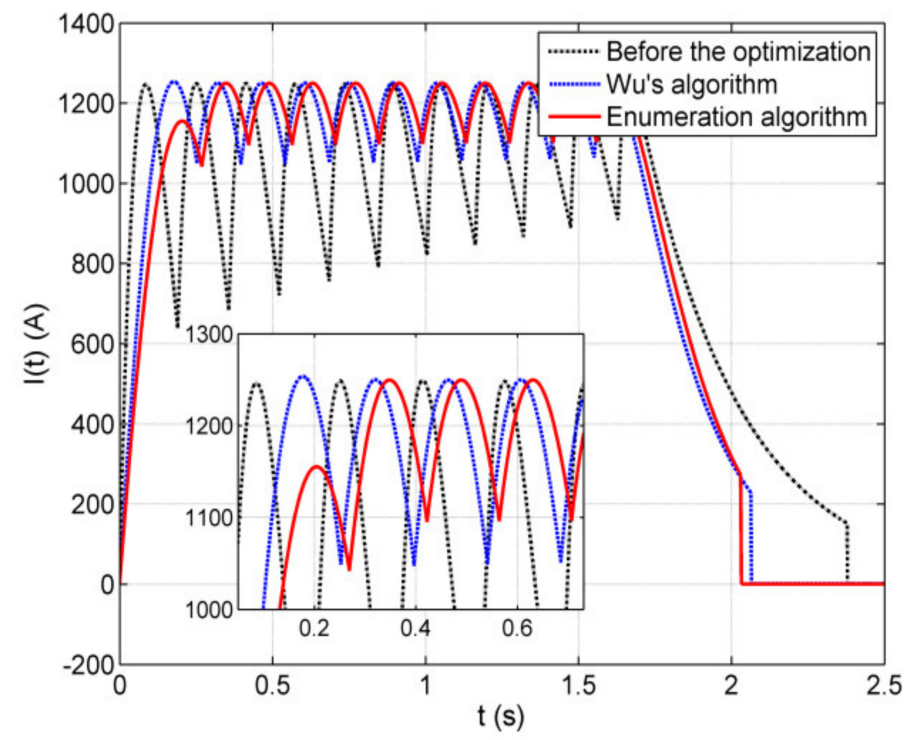

Figure 9. Charging current-time curves of the pulse capacitor of the two algorithms.

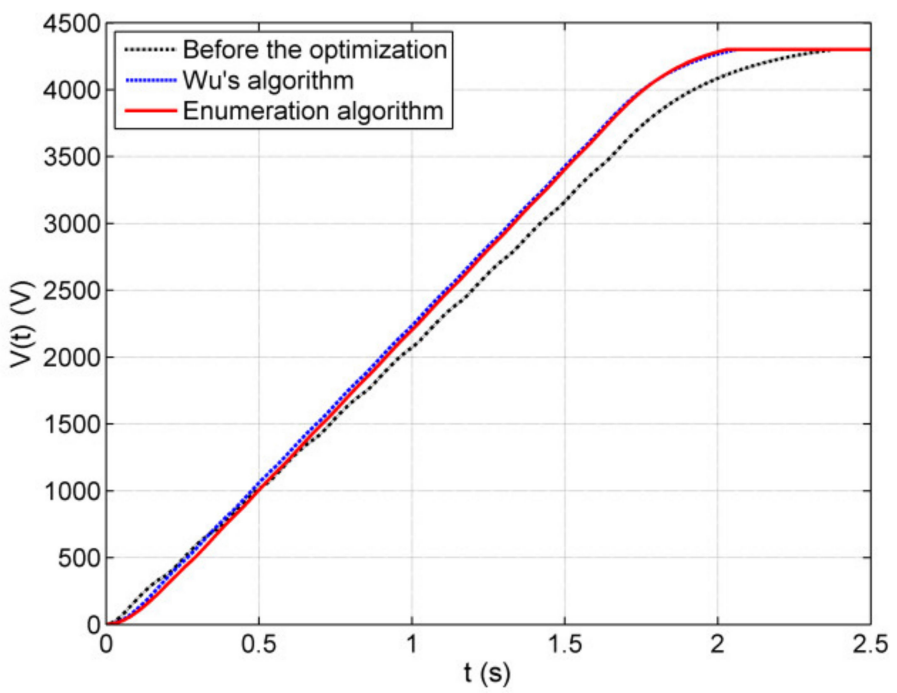

Figure 10. Charging voltage-time curves of the pulse capacitor of the two algorithms.

We also have studied the relationship between the first peak value of the charging current and the total charging time for all feasible solutions of the Enumeration algorithm (see Figure 11). Among these solutions, the minimum total charging time, with the constraint that the first peak value of the charging current needs to reach a specified value, ranks 59th if the charging time is arranged in ascending order. It is shown by the big red dot in Figure 11.

Figure 12 contains the plots of the relationship between the first peak value of the charging current and the total charging time for the two battery pack structures in the Enumeration algorithm's feasible solutions, respectively. Both results show that the corre- 
sponding solutions where the first peak value of the charging current is constrained are not the optimal solution.

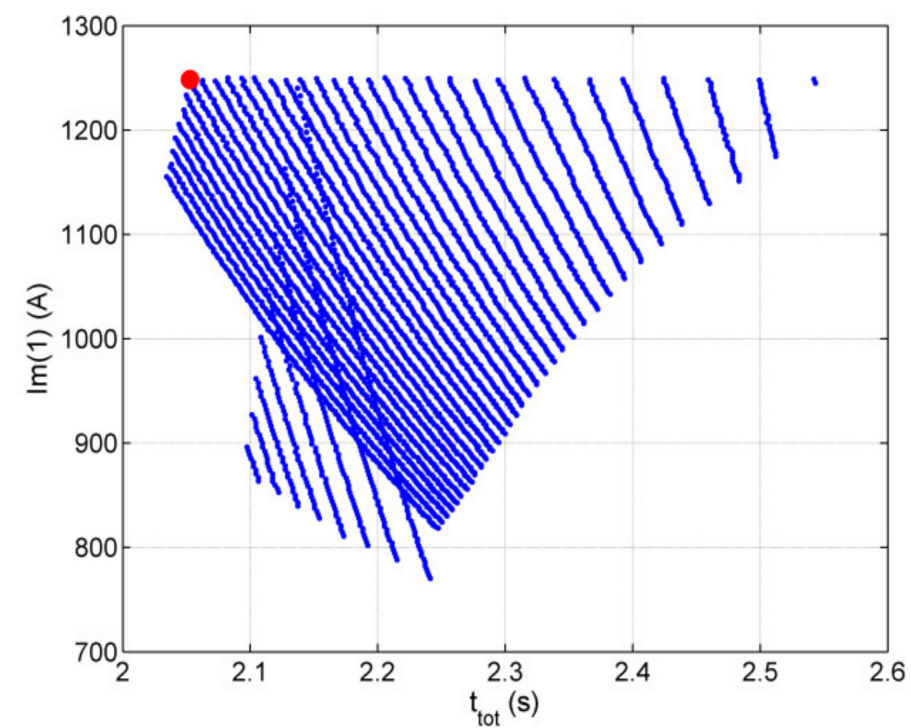

Figure 11. The relationship between the first peak value of the charging current and the total charging time for all feasible solutions of the Enumeration algorithm.

(a)

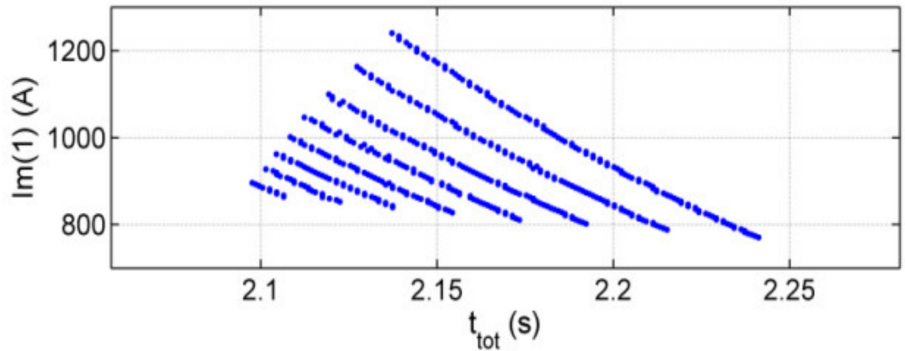

(b)

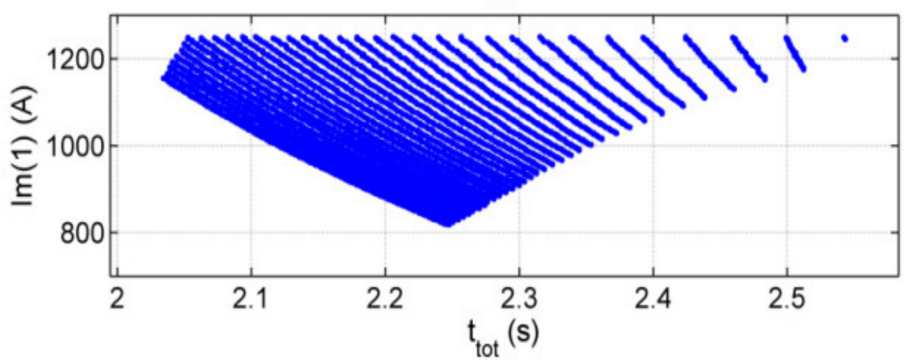

Figure 12. The relationship between the first peak value of the charging current and the total charging time for the two battery pack structures in the feasible solutions of the Enumeration algorithm, respectively. (a) $N=25$. (b) $N=11$.

Furthermore, the relationship between the total charging time and the time constant $\tau_{1}$ for the two battery pack structures in the feasible solutions of the Enumeration algorithm are presented in Figure 13, respectively. From the figures, the total charging time shows a decreasing trend, with the increase of the time constant $\tau_{1}$, for both battery pack structure conditions. 
(a)

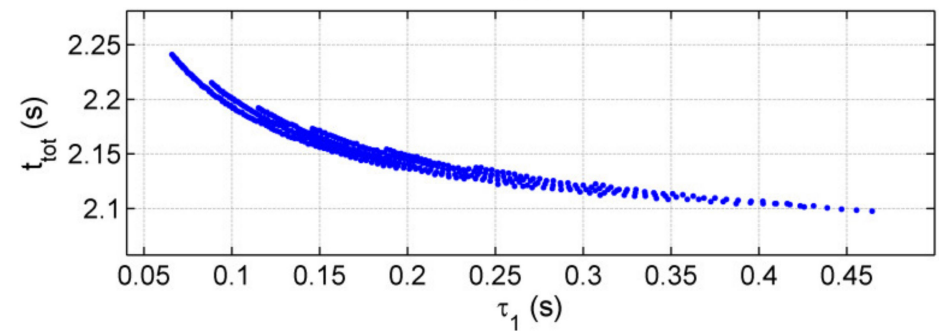

(b)

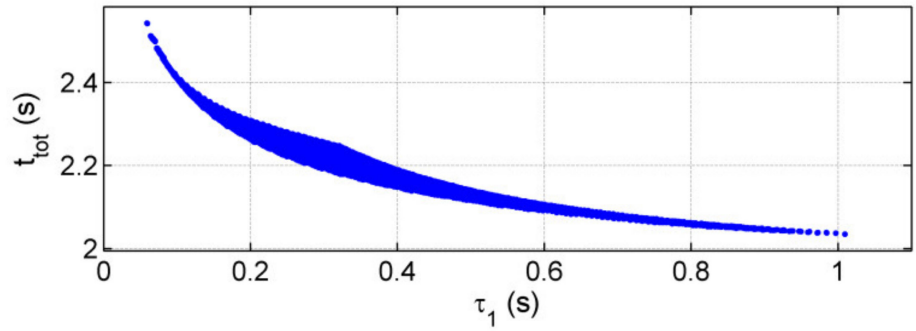

Figure 13. The relationship between the total charging time and time constant $\tau_{1}$ for the two battery pack structures in the feasible solutions of the Enumeration algorithm, respectively. (a) $N=25$. (b) $N=11$.

\subsection{Case 2}

Figure 14 shows the total charging time calculation results of all the Enumeration algorithm's feasible solutions. 6014 solutions in total. The pentagram shows the optimal solution.

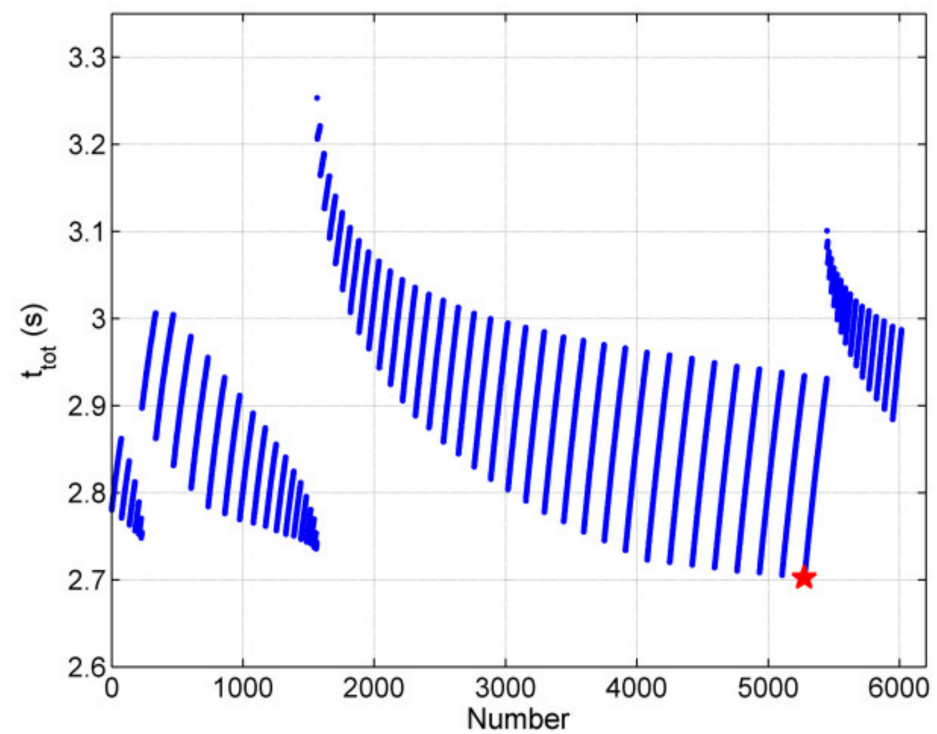

Figure 14. Calculation results of total charging time corresponding to all the variable vectors in $\mathbf{X}$.

Note that the results consist of four parts. They correspond to four battery pack structures, respectively. The details of the four battery pack structures are shown in Table 7.

Table 8 presents the comparison of the optimal solutions of both algorithms. The Enumeration algorithm outperforms the compared algorithm. The optimal solutions of both algorithms are much better than the solution before the optimization.

Moreover, the battery pack structure of the specific circuit that Wu's algorithm aims at is the same as that in the optimal solution of the Enumeration algorithm. Nevertheless, the solving process is unknown. This specific circuit has been optimized before the work in [22]. However, this process is not presented in the existing research. 
Table 7. Four battery pack structures in the solutions.

\begin{tabular}{cccccc}
\hline Condition & $\boldsymbol{n}_{\boldsymbol{s}}$ & $\boldsymbol{n}_{\boldsymbol{p}}$ & $\boldsymbol{N}$ & Number & $\boldsymbol{m i n}\left(\boldsymbol{t}_{\text {tot }}\right)(\mathbf{s})$ \\
\hline 1 & 50 & 5 & 35 & 231 & 2.75 \\
2 & 70 & 5 & 25 & 1332 & 2.74 \\
3 & 125 & 5 & 14 & 3882 & 2.70 \\
4 & 175 & 5 & 10 & 569 & 2.88 \\
\hline
\end{tabular}

Table 8. Comparison results of the two algorithms.

\begin{tabular}{ccccccc}
\hline & $n_{s}$ & $n_{p}$ & $N$ & $L(\mathbf{m H})$ & $R_{l}(\mathbf{m} \Omega)$ & $\min \left(t_{\text {tot }}\right)(\mathbf{s})$ \\
\hline Before the optimization & 125 & 5 & 14 & 11.5 & 152 & 3.08 \\
Wu's algorithm & 125 & 5 & 14 & 31.2 & 37 & 2.74 \\
Enumeration algorithm & 125 & 5 & 14 & 40 & 30 & 2.70 \\
\hline
\end{tabular}

Figures 15 and 16 show the comparison of the charging current-time and charging voltage-time curves of the two algorithms, respectively.

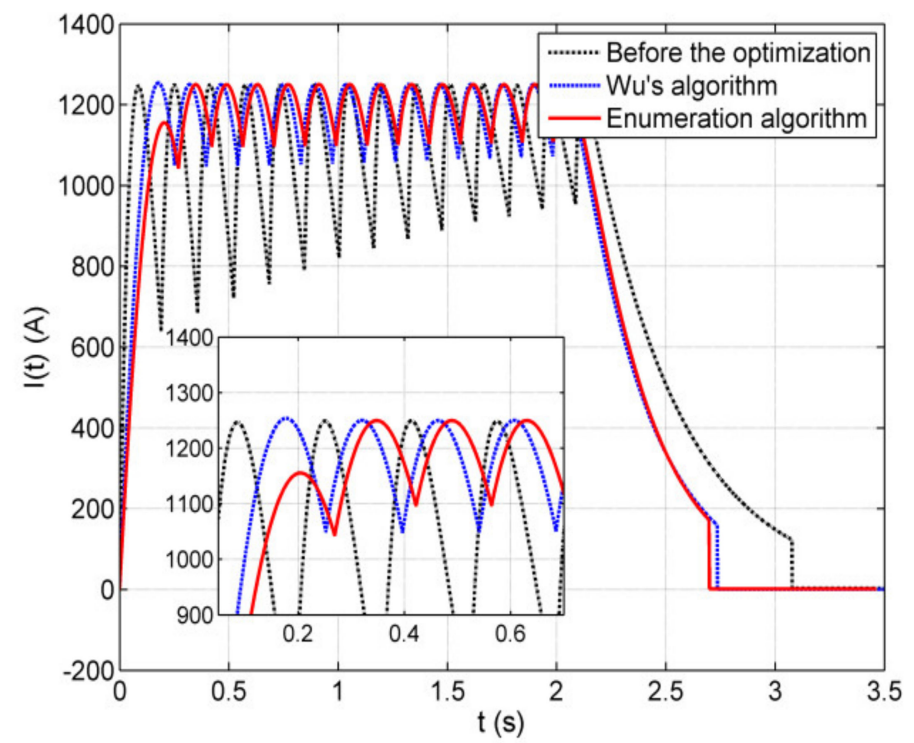

Figure 15. Charging current-time curves of the pulse capacitor of the two algorithms.

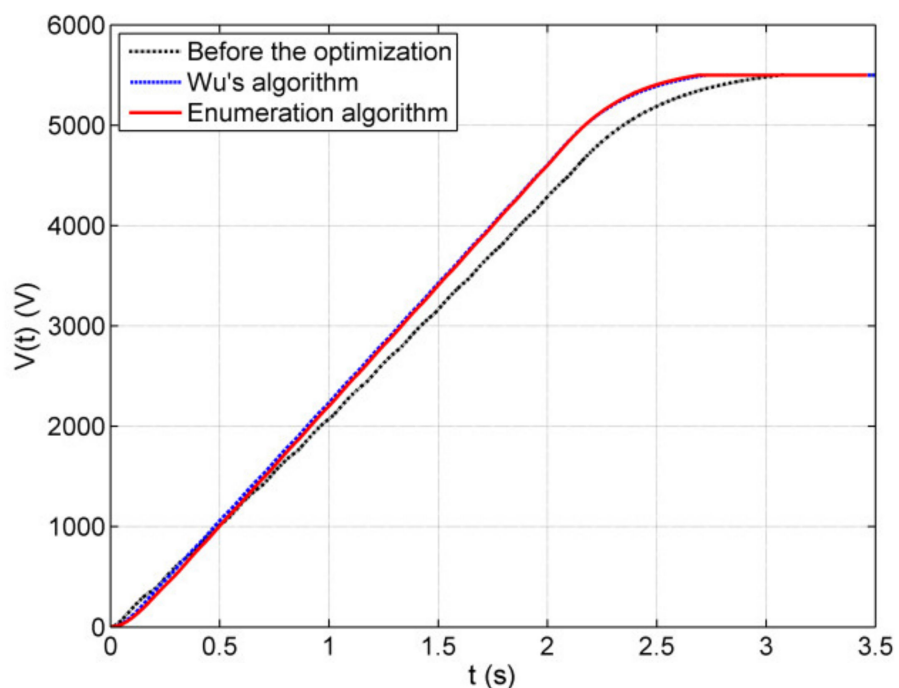

Figure 16. Charging voltage-time curves of the pulse capacitor of the two algorithms. 
As shown in Figure 15, the first peak value of the charging current is 1155 A for the Enumeration algorithm, and all the other peak values reach the required maximum charging current, $1250 \mathrm{~A}$. By contrast, all the peak values reach the required maximum charging current, $1250 \mathrm{~A}$, for both the "Wu's algorithm" and "before the optimization". Note that the charging current of the Enumeration algorithm has more minor fluctuations and is closer to a constant value than the compared algorithm. The total charging time of the Enumeration algorithm is slightly shorter than that of $\mathrm{Wu}^{\prime}$ 's algorithm, and obviously shorter than that of "before the optimization". Hence, in order to shorten the charging time, the constraint condition that the first peak current needs to reach a specified value is unnecessary. In particular, it increases the unnecessary design difficulty of the currentlimiting inductor.

Figure 16 shows the charging voltage curves. As can be seen from the figure, the charging voltage increases approximately linearly in the charging process. The charging speed of both algorithms is obviously faster than that before the optimization.

The relationship between the first peak value of the charging current and the total charging time for all feasible solutions of the Enumeration algorithm is shown in Figure 17. Note that the minimum total charging time with the constraint that the first peak value of the charging current needs to reach a specified value ranks 58th if the charging time is arranged in ascending order (see the big red dot in Figure 17).

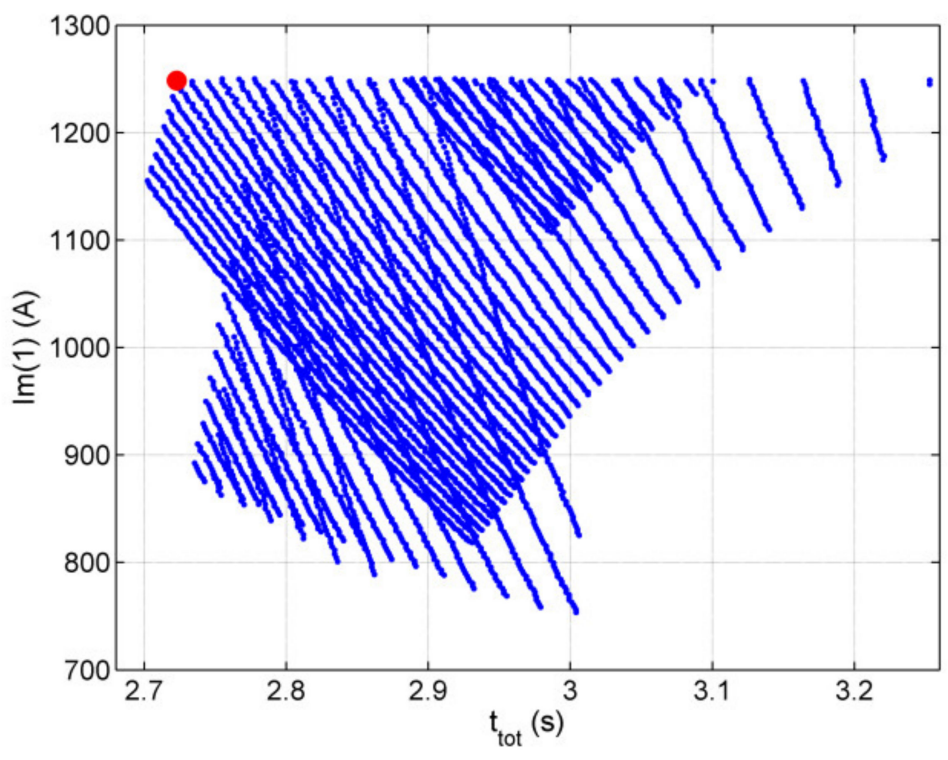

Figure 17. The relationship between the first peak value of the charging current and the total charging time for all feasible solutions of the Enumeration algorithm.

Figure 18 contains the plots of the relationship between the first peak value of the charging current and the total charging time for the four battery pack structures in the Enumeration algorithm's feasible solutions, respectively. The first three results show that the corresponding solutions, where the first peak value of the charging current is constrained, are not the optimal solution, and only the last result is an exception (see Figure 18d). It is just a coincidence.

The relationship between the total charging time and time constant $\tau_{1}$ for the four battery pack structures in the Enumeration algorithm's feasible solutions are presented in Figure 19, respectively. From the figures, the total charging time shows a decreasing trend with the time constant increase for all the battery pack structure conditions. 
(a)

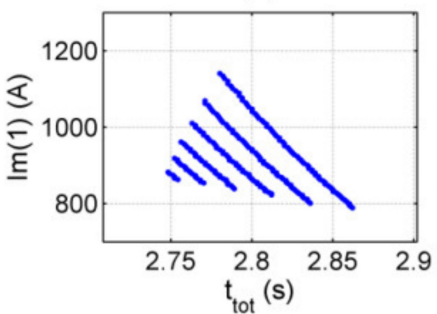

(c)

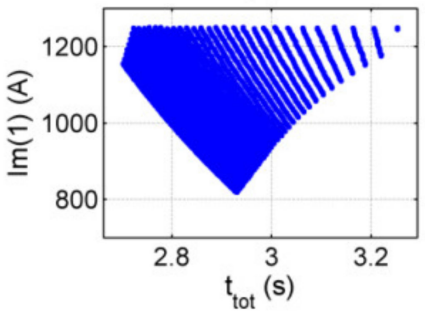

(b)

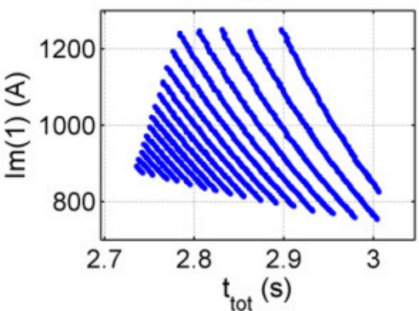

(d)

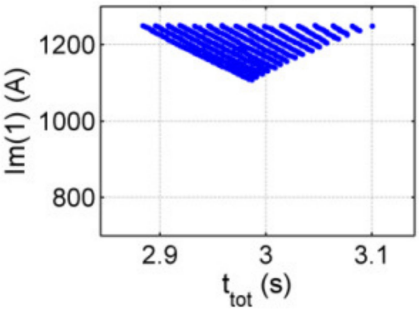

Figure 18. The relationship between the first peak value of the charging current and the total charging time for the four battery pack structures in the feasible solutions of the Enumeration algorithm, respectively. (a) $N=35$. (b) $N=25$. (c) $N=14$. (d) $N=10$.

(a)

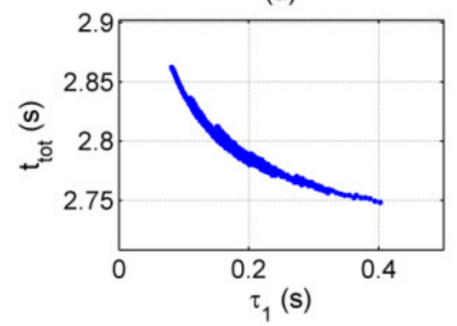

(c)

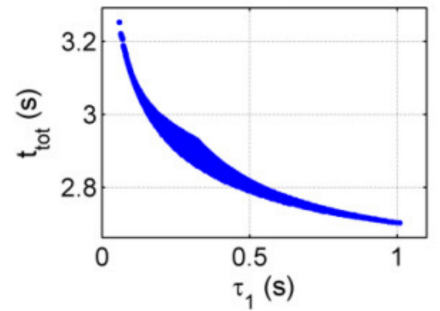

(b)

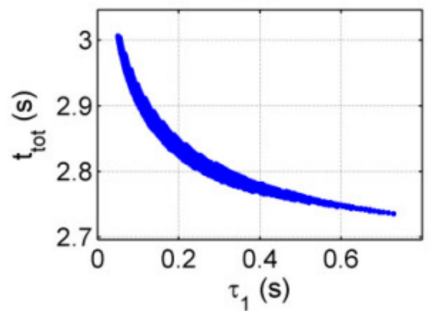

(d)

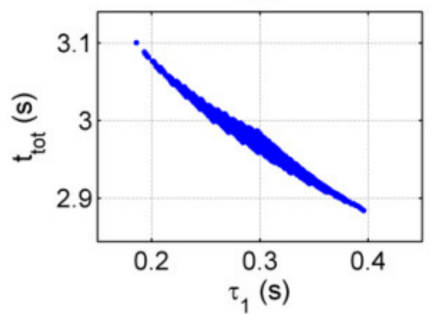

Figure 19. The relationship between the total charging time and time constant $\tau_{1}$ for the four battery pack structures in the Enumeration algorithm's feasible solutions, respectively. (a) $N=35$. (b) $N=25$. (c) $N=14$. (d) $N=10$.

\section{Conclusions}

In this paper, a novel optimization model has been constructed by considering the charging time problem of the hybrid energy storage system in the electromagnetic launch as a constrained optimization problem. It aims at an unknown circuit to be designed, cancels the unreasonable constraint on the first peak of the charging current, and finds the globally optimal solution through the Enumeration algorithm. Simulation studies have been carried out. In both cases, the simulations verified that the constraint condition of the first peak value of the charging current reaching a specified value limits the optimal design of the circuit and is unable to obtain a better solution. The proposed algorithm performs better than the compared algorithm. It makes the circuit design more flexible for the hybrid energy storage system, and particularly more flexible for the design of the current-limiting inductor in the circuit. 
It is worth noting that the total charging time of the proposed algorithm is slightly shorter than that of the compared algorithm, but not significantly. The battery pack structure of the specific circuit that $\mathrm{Wu}$ 's algorithm aims at is the same as that in the optimal solution of the Enumeration algorithm. Thus, we can see that this specific circuit has been optimized before using Wu's algorithm to optimize, but the optimization process is unknown. Therefore, the existing research is incomplete.

Furthermore, both cases obtain the same regularity: that the total charging time shows a decreasing trend with the time constant increase. Utilizing this regularity to optimize the circuit will be significant in our future research.

Author Contributions: Conceptualization, T.M. and J.L.; methodology, T.M.; software, T.M.; validation, X.Z. and W.W.; formal analysis, B.Z.; investigation, T.M.; resources, J.L.; data curation, X.L.; writing-original draft preparation, T.M.; writing-review and editing, B.Z.; visualization, T.M.; supervision, X.Z.; project administration, J.L.; funding acquisition, J.L. All authors have read and agreed to the published version of the manuscript.

Funding: This research was funded, in part, by the National Natural Science Foundation of China, grant number 51925704, 51877214, and 51907203.

Data Availability Statement: The data presented in this study are available on request from the corresponding author.

Conflicts of Interest: The authors declare no conflict of interest.

\section{References}

1. Fair, H.D. Progress in electromagnetic launch science and technology. IEEE Trans. Magn. 2007, 41, 93-98. [CrossRef]

2. McNab, I.R. Large-scale pulsed power opportunities and challenges. IEEE Trans. Plasma Sci. 2014, 42, 1118-1126. [CrossRef]

3. Marshall, R.A.; Wang, Y. Railguns: Their Science and Technology; China Machine Press: Beijing, China, 2004.

4. Yu, X.; Liu, X. Review of the Meat Grinder Circuits for Railguns. IEEE Trans. Plasma Sci. 2017, 45, 1086-1094. [CrossRef]

5. Yu, X.; Liu, X. Overview of Circuit Topologies for Inductive Pulsed Power Supplies. CES Trans. Electr. Mach. Syst. 2017, 1, 265-272. [CrossRef]

6. Liebfried, O. Review of Inductive Pulsed Power Generators for Railguns. IEEE Trans. Plasma Sci. 2017, 45, 1108-1114. [CrossRef]

7. Li, H.; Zhang, T.; Chen, Y.; An, Y.; Hu, Y.; Jiao, C. Feasibility Study of a Repetitive Inductive Pulsed Power Supply Circuit for Electromagnetic Rail-guns. IEEE Trans. Appl. Supercond. 2018, 29, 5000306. [CrossRef]

8. Tao, X.; Liu, K.; Wang, H. Modeling of an Air-Core Compulsator and the Pulse Shaping Using Optimization Algorithm. IEEE Trans. Plasma Sci. 2019, 47, 611-618. [CrossRef]

9. Consolo, V.; Musolino, A.; Rizzo, R.; Sani, L. Numerical 3D Simulation of a Full System Air Core Compulsator-Electromagnetic Rail Launcher. Appl. Sci. 2020, 10, 5903. [CrossRef]

10. Ye, C.; Li, W.; Yang, J.; Chang, S.; Zhi, G.; Xiang, Y.; Zhu, D. Development and Analysis of a Novel Cascaded Br ushless Self-Excited Air-Core Compensated Pulsed Alter nator With Squirrel-Cage Rotor Winding. IEEE Trans. Ind. Electron. 2021, 68, 5571-5581. [CrossRef]

11. Sitzman, A.; Surls, D.; Mallick, J. Design, Construction, and Testing of an Inductive Pulsed-Power Supply for a Small Railgun. IEEE Trans. Magn. 2007, 43, 270-274. [CrossRef]

12. Dedie, P.; Brommer, V.; Scharnholz, S. Twenty-stage toroidal XRAM generator switched by countercurrent thyristors. IEEE Trans. Plasma Sci. 2011, 39, 263-267. [CrossRef]

13. Dierks, E.; McNab, I.R.; Mallick, J.A., Sr.; Fish, S. Battery Inductor Parametric System Analysis for Electromagnetic Guns. IEEE Trans. Plasma Sci. 2011, 39, 268-274. [CrossRef]

14. Sitzman, A.; Surls, D.; Mallick, J. Modification and testing of a battery-inductor repetitive pulsed power supply for a small railgun. In Proceedings of the 2007 16th IEEE International Pulsed Power Conference, Albuquerque, NM, USA, 17-22 June 2007; pp. 1793-1798.

15. Dong, J.; Li, J.; Gui, Y.; Deng, Q.; Peng, Y. A 600kJ Pulsed Power Supply for Electromagnetic Launching Application. In Proceedings of the 2005 IEEE Pulsed Power Conference, Monterey, CA, USA, 13-15 June 2005; pp. $108-111$.

16. Ma, W.; Lu, J. Thinking and Study of Electromagnetic Launch Technology. IEEE Trans. Plasma Sci. 2017, 45, 1071-1077. [CrossRef]

17. Ma, W.; Lu, J.; Liu, Y. Research Progress of Electromagnetic Launch Technology. IEEE Trans. Plasma Sci. 2019, 47, 2197-2205. [CrossRef]

18. Allen, R.J.; Boyer, C.N.; Neri, J.M.; Veracka, M. Development of A 150-kW, Battery Powered, Rapid Capacitor Charger For A Small Railgun in Burst Mode Operation at 3 RPS. In Proceedings of the 2006 IEEE International Power Modulators and High Voltage Conference, Las Vegas, NV, USA, 27-31 May 2008; pp. 106-108.

19. Liu, K.; Gao, Y.; Fu, R.; Sun, Y.; Yan, P. Design of Control System for Battery Cascade Charging Power Supply. IEEE Trans. Plasma Sci. 2017, 45, 1245-1250. [CrossRef] 
20. Long, X.; Lu, J.; Zhang, X.; Zhou, R.; Xiong, Y. Double Loop Control Method for Hybrid Energy Storage System. J. Nav. Univ. Eng. 2016, 28, 13-16.

21. Li, C.; Lu, J.; Jiang, H.; Long, X.; Zheng, Y. Comparison of Charging Methods of Multilevel Hybrid Energy Storage for Electromagnetic Launch. High Power Laser Part. Beams 2015, 27, 0750051-0750056.

22. Wu, Y.; Lu, J.; Long, X.; Zhou, R.; Liu, Y. Optimization of Energy Transfer Rate of Hybrid Energy Storage System in Electromagnetic Launch. High Volt. Eng. 2019, 45, 3715-3720. 